\title{
Corrective exercises administered online vs at the workplace for pain and function in the office workers with upper crossed syndrome: Randomized controlled trial
}

\section{Zohreh Yaghoubitajani}

Shahid Beheshti University

Mehdi Gheitasi ( $\square$ ariana.zohre.amir@gmail.com )

Shahid Beheshti University https://orcid.org/0000-0002-6652-8567

Mohammad Bayattork

Hormozgan University

Lars Louis Andersen

National Research Centre

\section{Research Article}

Keywords: Online-Supervised, Workplace Corrective Exercises, Neck-shoulder Pain, Workability, Muscle Activation, Postural malalignment

Posted Date: January 25th, 2022

DOI: https://doi.org/10.21203/rs.3.rs-1257973/v1

License: (c) (1) This work is licensed under a Creative Commons Attribution 4.0 International License. Read Full License

Version of Record: A version of this preprint was published at International Archives of Occupational and Environmental Health on April 7th, 2022. See the published version at https://doi.org/10.1007/s00420022-01859-3. 


\section{Abstract \\ Objective}

To evaluate the effects of online-supervised versus workplace corrective exercises on neck-shoulder pain (NSP), sick-leave, postural angles, workability, and electromyography (EMG) of interest muscles among the office workers with the upper crossed syndrome (UCS).

\section{Methods}

We performed a parallel-group randomized control trial at Shahid Beheshti University (SBU), Tehran, Iran. The office workers $(n=36)$ were randomly assigned to online-supervised, workplace, and control groups (each: $n=12$ ). The two intervention groups completed the exercise program for eight weeks, while the control group continued usual activities. Primary (NSP and sick-leave) and secondary outcomes (forward head posture, round shoulders, and round back angles), workability, EMG (upper, middle, lower trapezius, sternocleidomastoid, and serratus anterior) were measured using visual analog scale (VAS), questionnaire, photogrammetry, and EMG, respectively. All measurements were performed at the baseline and an 8-week follow-up.

\section{Results}

In both intervention groups, paired t-tests results demonstrated significant differences in NSP, forward head posture, round shoulders, and round back, respectively. In the online-supervised, workability (physical and mental) improved, and upper trapezius and serratus anterior differed in Max., Ave., activation, offset timing changed in the upper trapezius, respectively. Using ANCOVA, the onlinesupervised group revealed significant differences, including NSP, postural angles, physical and mental workability, and upper trapezius muscle activation. The sick-leave and other variables were not the cases in all groups.

\section{Conclusion}

Online-supervised corrective exercise seems to improve a range of parameters related to work performance. These findings are highly applicable in light of the ongoing COVID pandemic; many workers have to work from home.

\section{Introduction}

Some researchers have proposed that prolonged static postures - e.g. sitting in the same forward-leaned position in front of a computer all the day - may cause postural malalignment and be considered as a risk factor for work-related musculoskeletal disorders (WMSDs) (Szeto, Straker et al. 2005). Janda believed 
that sustained awkward positions could very likely lead to transformation in the head, shoulder, and spinal curves, causing forward head posture, round shoulders, and round back, anteriorly tilt, and winged scapula(Zad and Patil 2021). Such conditions are postulated to result in constant pressure and degenerative changes in individual's joints and are simultaneously associated with musculoskeletal imbalance and pain (Isper Garbin, Soares et al. 2017). Based on the literature, neck and shoulder impairments, headaches, chronic neck tension, and scapular dyskinesia could negatively influence the workability and typical daily performance (Singla and Veqar 2017). Furthermore, many studies have reported the prevalence rates of approximately $50.5 \%$ and $44.8 \%$ shoulder and upper back pain among the office workers (Madeleine, Lundager et al. 1999). In 2014, Nejati et al. published a high prevalence of UCS symptoms containing forward head posture, round shoulders, and round back as 61.3, 48.7, and $78.3 \%$, respectively among Iranian office workers (Nejati, Lotfian et al. 2014). However, high-quality research based on prospective cohort studies found no association between the computer use and the risk of chronic neck and shoulder pain (Andersen, Harhoff et al. 2008). Thus, it is why only certain individuals are prone to develop UCS. The previous research have approved that increasing pain intensityregardless of its cause, decreases the workability among sedentary and physical work demands (Bayattork, Jakobsen et al. 2019). Due to musculoskeletal symptoms imposing indirect costs including sick-leave, productivity loss, treatment costs, disability and work absenteeism in office workers, the issues such as lifestyle interventions, identification of the groups at the risk of disability, increased health promotion and early prevention in the workplace could be assumed as great potential for increasing workability and reducing work absenteeism (Sundstrup, Seeberg et al. 2020).

Additionally, UCS is characterized by tightening upper trapezius and sternocleidomastoid following the deep cervical flexors and middle, lower trapezius, and serratus anterior muscles' weakness (Ohlendorf, Erbe et al. 2017). It may cause alteration in scapula muscle activation, movement patterns, and some misalignments in the upper limb that simultaneously contribute to cervicothoracic and glenohumeral joint dysfunction (Seidi, Bayattork et al. 2020). Acting as a bridge between the shoulder and cervical region, the scapula plays a significant role in providing mobility and stability for the neck and shoulders (Cools, Struyf et al. 2014). Previous studies also indicated the relationship between chronic NSP and scapular dyskinesis through altering dynamic scapular stability during the scapular orientation (Cagnie, Struyf et al. 2014). Numerous studies have determined impaired muscle coordination in the neck muscles, cervical pain, and abnormal head position associated with high amounts of EMG activity in the neck flexors' muscles and have highlighted a direct correlation among upper trapezius muscle activity, neck angle, and perceived discomfort during the prolonged computer tasks (Falla, Jull et al. 2004, D'Anna, Schmid et al. 2021). Brandt et al. reported a strong association between perceived NSP intensity and trapezius muscle tenderness among the office workers (Brandt, Sundstrup et al. 2014).

Based on the previous findings, serratus anterior and lower trapezius provide stability for the scapula and maintain the appropriate scapular location to act as an essential shoulder stabilizer and balance the scapular rotation coupling forces (Johnson, Bogduk et al. 1994). Consequently, altered stability of the scapula may create or sustain symptomatic mechanical dysfunction in the cervical spine and influence the neck pain's recurrence (Helgadottir, Kristjansson et al. 2011). Meanwhile, disturbances in scapular 
function could be found in both shoulder and neck pain due to the neuromuscular imbalance between the deep phasic and superficial tonic muscles. This phenomenon is worthwhile to be considered for effective management of UCS among the office workers(Page, Frank et al. 2010). In this regard, Cools et al. suggested basic rehabilitation approach, which is focused on the office workers' functional demands, like correcting the function of axioscapular muscles and scapular alignment during the prolonged upper extremities activities (Cools, Struyf et al. 2014). Hence, implementing posture-correction strategies early in the rehabilitation program, performing all interventions in a sitting or standing position with a correct posture, and maintaining an upright body position to retrain a neutral posture, are considered as different techniques for reduction of the chronic neck pain (Falla, Jull et al. 2007).

Although, several studies investigated the efficiency of workplace interventions for rehabilitation or prevention of WMSDs as a global health issue,which influence both employers and employees, an effective intervention is still needs to be warranted (Skamagki, King et al. 2018). Due to lack of research on computer users suffering from UCS, it seems logical to evaluate associated various musculoskeletal symptoms, including forward head posture, round shoulders, and round back along with related muscles' EMG influenced by UCS, and pain in neck and shoulder areas, as well as assessing the extent of workability and sick-leave variables in such populations. Although, most research reported positive effects of supervised and un-supervised interventions in managing WMSDs, some studies underlined that supervised exercise programs are most likely to be beneficial in maintenance and adherence. As a result, they have significantly better influence on diminishing consequential symptoms (Blangsted, Søgaard et al. 2008, Coury, Moreira et al. 2009). Conversely, implementing exercise under supervision can be costeffective and not always an available workplace resource; however, some individuals prefer to exercise when it fits into their daily working routine (Gram, Andersen et al. 2014). Since many countries worldwide have applied social quarantine during the COVID-19 global pandemic, the office workers were advised to work from home and follow the rules of social distance (Aegerter, Deforth et al. 2021). Nevertheless, evidence-based guidelines for performing home-exercise are lacking. The current study is the first randomized controlled trial with the purpose of comparing the effect of corrective exercises at the worksite versus home under direct supervision during the pandemic.

In more detail, the primary objectives were to assess the effects of an eight-week online-supervised homebased versus workplace-based corrective exercises to decrease NSP and sick-leave. The secondary objectives were to evaluate postural angles (forward head posture, round shoulders, and round back), workability, the surface EMG of interest muscles (upper, middle, lower trapezius, sternocleidomastoid, and serratus anterior) among the computer users working at the offices with UCS.

\section{Methods}

\section{Study Design,Participants}

A parallel-group randomized controlled trial with testing at baseline and follow-up was approved by Ethics' Committee on the Research at SBU, Tehran, Iran (approval number: IR.SBU.REC.1399.036 / 
2020.06.20). The trial was registered at IRCT (IRCT20200729048249N1 / 2020.10.05). The assessments were conducted at baseline and after eight weeks of intervention in the Sport Sciences and Health Laboratory at SBU, Tehran, Iran. The subjects signed an informed consent followed by the Declaration of Helsinki. They were randomly assigned to three groups, the intervention groups (online-supervised and workplace) and the control group. Fig. 1 demonstrates the whole procedure according to CONSORT guidelines in order to ensure the transparent and standardized reporting of the trial. The study protocol has been published, and all detailed procedures have been already described elsewhere (Yaghoubitajani, Gheitasi et al. 2021).

The entire study procedure, including assessments, was clarified for 118 volunteers who were invited through invitation letters. Qualified office workers, including 23 females and 13 males, were performed the pre-test and they were all able to meet the eligibility criteria with postural alterations (forward head posture $\geq 45^{\circ}$, round shoulders $\geq 52^{\circ}$, round back $\left.\geq 42^{\circ}\right)$ and pain intensity $(\geq 3)$ in the neck and shoulder areas, measured by photogrammetry and VAS, respectively. Based on the above inclusion criteria, the individuals were excluded from the list of participants if they were pregnant, had a history of joint diseases in the spine, shoulder, pelvis, fracture, and had surgery during the past year, or had a bodyweight out of the normal range ( $18 \geq \mathrm{BMI} \geq 25)$. The subjects were considered as drop-outs if they missed three sequential sessions or the follow-up test. For their conveniences, the subjects were permitted to leave the study at any stage of the research process.

\section{Study Outcomes, Assessments}

The chief researcher measured the study outcomes at the baseline before randomization and after eight weeks. The NSP and sick-leave as the primary outcomes were measured by VAS and questionnaire. The secondary outcomes, including postural alignment angles (forward head posture, round shoulders , and round back), workability (physical and mental), muscle activation, and timing of the interested muscles (upper, middle, lower trapezius, sternocleidomastoid, and serratus anterior), were assessed by photogrammetry, questionnaire, and EMG, respectively. Demographic data were obtained, including age, weight, height, BMI, smoking behavior, marital status, and education level. The baseline and follow-up test were conducted using assessment methods and testing equipment in the same setting.

Using a 10-cm VAS scale, the subjects rated their NSP intensity, ranging from zero ("no pain") to ten ("worst pain"). The sick-leave was assessed by a single item extracted fromevaluation questionnaire of validated outcome. The response categories ranged from zero to 31 days, inserting the number of absence working days due to NSP within the past month (Hallman, Holtermann et al. 2019). The photogrammetric method was applied for the postural angles' assessments by identifying anatomical landmarks of the tragus, acromion process of the scapula, spinous process of C-7 and T-12 through removable colored dots to be laterally visible (Karimian, Rahnama et al. 2019). The workability was evaluated by self-assessing through a single validated item from the workability index questionnaire. The scores range from zero to ten, indicating a cut-off point score of $\leq 7$, implying the poor workability (Gram, Holtermann et al. 2012, Hallman, Holtermann et al. 2019). 
The EMG recordings were made through the standard procedure to normalize the recommended range in work-related disorder studies for activation and timing of upper, middle, lower trapezius, sternocleidomastoid, and serratus anterior muscles following careful skin preparation based on SENIAM and previously published guidelines concerning the skin impedance $(\leq 10 \mathrm{k} \Omega$ ) and bipolar $\mathrm{Ag} / \mathrm{AgCl}$ (SKINTACT, Austria) surface electrodes' application (Johnston, Jull et al. 2008). The ME-6000 Megawin (MegaWin, Finland) was used for data collection, and all EMG signals were amplified (gain, 1000), passed through a 10 to $500 \mathrm{~Hz}$ band-width filter, and sampled at $1000 \mathrm{~Hz}$ processed through the Matlab software (Seidi, Bayattork et al. 2020). The maximal voluntary isometric test (MVIC) was used and normalized the EMG data. After training and a short warm-up, the subjects performed three MVICs holdings for five seconds against the manual static resistance, in which the duration was controlled by a stopwatch. Those subjects who reported pain during the test were excluded to minimize the impact of pain on the assessment consisting of a 30 seconds rest time between each repetition and two minutes for subsequent muscle testing. After five minutes of resting, the subjects performed bilateral arm elevation five times in the scapular plane over concentric, isometric, and eccentric phases, which lasted three seconds as well as three seconds of rest between each phase. After rectifying and smoothing, the root mean square (RMS) was calculated at the time constant of $50 \mathrm{~ms}$ for three seconds from the middle of three trials out of five. The RMS mean was divided using MVIC value, was multiplied by 100 to calculate the muscle activity percentage (Arshadi, Ghasemi et al. 2019). The muscle activation onset/offset was calculated based on concentric/eccentric phases from the point that the level of muscle activity reached three standard deviations above/below the rest of the muscle activity and then was analyzed based on the middle deltoid onset/offset timing (De Mey, Danneels et al. 2012).

\section{Randomization}

The computer-generated block randomization was performed in a 1:1:1 allocation ratio through the website https://www.sealedenvelope.com. The main investigator performed the enrollment, generated the random allocation sequence, and assigned the subjects to the groups. The random allocation sequence was implemented by concealed, sequentially numbered, sealed, and opaque envelopes, through putting a card inside indicating that the allocated group to each subject was randomly executed. A SBU assistant professor supervised all procedures, including the sequence generation process and allocation concealment mechanism to ensure that the assignment schedule was unpredictable.

\section{Intervention}

Intervention groups performed eight-week corrective exercises, three times/week, lasting about 50-60 minutes pertaining typical five-minute warm-up and cool-down. Each exercise was individualized based on demographic characteristics, pain intensity, and overload principles. The pain intensity up to five (VAS) was allowed to have ten max. repetition, only if the pain decreased immediately after completing the exercise. The subjects who experienced the higher pain levels, could modify the exercise accordingly.

The online-supervised group performed the program under direct remote supervision in their home environment using desktop videoconferencing software (https://meet.jit.si/). To do so, a laptop 
monitored by a qualified corrective exercises' expert was utilized to ensure the progress movement pattern and the control load progression. The workplace group who performed the program in their worksite were provided a diary with detailed written and pictorial descriptions. In addition to telephone interviews, expert's weekly presence was accentuated to constantly evaluate the progress, safety, and performance.

The exercise program was prescribed concerning spontaneous postural alterations, muscle activation, movement pattern, and lack of scapula stabilization for the individuals with UCS suffering from NSP. Detailed intervention is available elsewhere (Yaghoubitajani, Gheitasi et al. 2021).

\section{Sample Size and Statistical Analysis}

The G*Power software (Version 3.0.10, Germany) was used to calculate the sample size regarding the effect size reported for the corrective exercises compared with the control group without any intervention in a similar study $(n=33)$, however, 12 subjects were assigned in each group in order to avoid potential dropout during the research. The analyses were conducted using IBM SPSS version 20 for Windows (SPSS Inc., Chicago, IL, USA). Variables' distribution were examined by the Shapiro-Wilk ( $P \geq 0.05)$. Paired $\mathrm{t}$-test and one-way ANCOVA were used for within-group and between-group comparisons. The follow-up values were used as the outcome, while controlling the baseline values. The $M \pm S D$ and the significance levels at $p \leq 0.05$ were applied for data demonstration. The partial $\eta 2$ method was utilized to determine the effect size of the differences' magnitude as small $(0.01 \leq \eta 2<0.06)$, medium $(0.06 \leq \eta 2<0.14)$ or large ( $\eta 2 \geq 0.14)$ (Richardson 2011). For the minimum clinically important differences, the formula of $(M C I D=S D \times 0.5)$ was used (Norman, Sloan et al. 2003).

\section{Results}

The assessments were performed among 36 subjects, and the study outcomes were analyzed for the original assigned three groups. Table 1 demonstrates baseline demographic and lifestyle characteristics. All variables had normal distribution based on Shapiro-Wilk test $(P \geq 0.05)$. Tables 2 and 3 display withingroup and between-group differences, the magnitude effect sizes in study outcomes' improvement at the follow-up, and the MCIDs changes in the intervention groups, respectively. 
Table 1

demographic characteristics and lifestyle characteristics: mean \pm Standard deviation $(M \pm S D)$, and normality distribution

\begin{tabular}{|c|c|c|c|c|c|}
\hline Variable & Group & $\mathrm{M} \pm \mathrm{SD}$ & $\mathrm{t}$ & $\mathbf{p}$ & Percent(\%) \\
\hline \multirow[t]{3}{*}{ Age(year) } & Control & $38.91 \pm 3.87$ & 0.902 & 0.196 & \\
\hline & Online-supervised & $38.58 \pm 7.34$ & 0.941 & 0.517 & \\
\hline & Workplace & $37.00 \pm 8.12$ & 0.923 & 0.451 & \\
\hline \multirow[t]{3}{*}{ Weigh(kg) } & Control & $67.18 \pm 13.02$ & 0.878 & 0.099 & \\
\hline & Online-supervised & $66.33 \pm 8.97$ & 0.841 & 0.059 & \\
\hline & Workplace & $67.63 \pm 9.68$ & 0.953 & 0.746 & \\
\hline \multirow[t]{3}{*}{ Height(m) } & Control & $1.72 \pm 0.09$ & 0.959 & 0.756 & \\
\hline & Online-supervised & $1.67 \pm 0.08$ & 0.913 & 0.235 & \\
\hline & Workplace & $1.74 \pm 0.07$ & 0.894 & 0.253 & \\
\hline \multirow[t]{3}{*}{$\mathrm{BMI}\left(\mathrm{kg} / \mathrm{m}^{2}\right)$} & Control & $24.27 \pm 2.91$ & 0.919 & 0.310 & \\
\hline & Online-supervised & $25.86 \pm 1.77$ & 0.919 & 0.281 & \\
\hline & Workplace & $25.90 \pm 1.82$ & 0.936 & 0.570 & \\
\hline \multirow[t]{3}{*}{ Smoking } & \multicolumn{4}{|l|}{ Control(Yes/No) } & 27.3/72.7 \\
\hline & \multicolumn{4}{|c|}{ Online-supervised (Yes/No) } & $0.0 / 100.0$ \\
\hline & \multicolumn{4}{|l|}{ Workplace (Yes/No) } & $25.0 / 75.0$ \\
\hline \multirow[t]{3}{*}{ Marital Status } & \multicolumn{4}{|c|}{ Control (single/married) } & $18.2 / 81.8$ \\
\hline & \multicolumn{4}{|c|}{ Online-supervised (single/married) } & $66.7 / 33.3$ \\
\hline & \multicolumn{4}{|c|}{ Workplace (single/married) } & $50.0 / 50.0$ \\
\hline \multirow[t]{3}{*}{ Educational Level } & \multicolumn{4}{|c|}{ Control (BS, MSc, Ph.D) } & $45.5 / 27.3 / 27.3$ \\
\hline & \multicolumn{4}{|c|}{ Online-supervised (Diploma, BS, MSc) } & $16.7 / 33.3 / 50.0$ \\
\hline & \multicolumn{4}{|c|}{ Workplace (Diploma, BS, MSc) } & $12.5 / 62.5 / 25.0$ \\
\hline
\end{tabular}


Table 2

Within-group differences (Paired T-Test) in NSP; Sick-leave(SL); Workability (WA)physical/mental; Forward head posture(FHP), Round shoulders(RSH), Round back(RB) angles; ave./ max. activity, onset/offset of Upper, Middle, Lower trapezius, Serratus anterior, and Sternocleidomastoid(UT,MT,LT,SA, and SCM).

\begin{tabular}{|c|c|c|c|c|c|c|}
\hline \multirow[t]{2}{*}{ Group } & \multirow{2}{*}{$\begin{array}{l}\text { Variable } \\
\text { (baseline/ } \\
\text { follow-up) }\end{array}$} & \multirow[t]{2}{*}{$\begin{array}{l}\text { Mean } \\
\text { Difference }\end{array}$} & \multicolumn{2}{|c|}{$\begin{array}{l}95 \% \text { Confidence Interval of the } \\
\text { Difference }\end{array}$} & \multirow[t]{2}{*}{$\mathrm{t}$} & \multirow[t]{2}{*}{ Sig. } \\
\hline & & & Lower & Upper & & \\
\hline \multirow[t]{24}{*}{ Control } & NSP & 0.45455 & -0.00736 & 0.91645 & 2.193 & 0.053 \\
\hline & $S L$ & 0.09091 & -0.11165 & 0.29347 & 1.000 & 0.341 \\
\hline & WA.physical & 0.27273 & -0.25548 & 0.80093 & 1.150 & 0.277 \\
\hline & WA.mental & -0.09091 & -0.45326 & 0.27144 & -0.559 & 0.588 \\
\hline & FHP & 0.04545 & -0.30539 & 0.39630 & 0.289 & 0.779 \\
\hline & $\mathrm{RSH}$ & 0.00909 & -1.53766 & 1.55584 & 0.013 & 0.990 \\
\hline & $\mathrm{RB}$ & -0.23636 & -1.48682 & 1.01409 & -0.421 & 0.683 \\
\hline & UT - ave. & -0.03182 & -0.09037 & 0.02673 & -1.211 & 0.254 \\
\hline & UT - max. & -0.07091 & -0.15618 & 0.01436 & -1.853 & 0.094 \\
\hline & UT - onset & -209.754 & -641.00996 & 221.50087 & -1.084 & 0.304 \\
\hline & UT - offset & -36.5727 & -1372.7919 & 1299.6465 & -0.061 & 0.953 \\
\hline & MT - ave. & -0.08818 & -0.17138 & -0.00498 & -2.362 & 0.060 \\
\hline & MT - max. & -0.20636 & -0.40210 & -0.01063 & -2.349 & 0.061 \\
\hline & MT - onse & -238.454 & -595.92326 & 119.01417 & -1.486 & 0.168 \\
\hline & MT - offset & 1022.663 & -456.49804 & 2501.8253 & 1.540 & 0.154 \\
\hline & LT - ave. & -0.07091 & -0.15878 & 0.01696 & -1.798 & 0.102 \\
\hline & LT - max. & -0.18909 & -0.39920 & 0.02101 & -2.005 & 0.073 \\
\hline & LT - onset & -73.3090 & -433.51906 & 286.90088 & -0.453 & 0.660 \\
\hline & LT - offset & 231.5727 & -1017.1562 & 1480.3017 & 0.413 & 0.688 \\
\hline & SA - ave. & -0.05818 & -0.14805 & 0.03169 & -1.442 & 0.180 \\
\hline & SA - max. & -0.11364 & -0.29595 & 0.06868 & -1.389 & 0.195 \\
\hline & SA - onset & 39.80909 & -417.37975 & 496.99793 & 0.194 & 0.850 \\
\hline & SA- offset & 312.0454 & -558.06528 & 1182.1561 & 0.799 & 0.443 \\
\hline & SCM - ave. & -0.05455 & -0.11038 & 0.00129 & -2.177 & 0.055 \\
\hline
\end{tabular}




\begin{tabular}{|c|c|c|c|c|c|c|}
\hline & SCM -max. & -0.13545 & -0.27971 & 0.00880 & -2.092 & 0.063 \\
\hline & SCM - onset & 317.2636 & -621.44766 & 1255.9749 & 0.753 & 0.469 \\
\hline & SCM - offset & 139.2818 & -1120.7945 & 1399.3581 & 0.246 & 0.810 \\
\hline \multirow{26}{*}{$\begin{array}{l}\text { Online- } \\
\text { supervised }\end{array}$} & NSP & 3.91667 & 2.52310 & 5.31023 & 6.186 & $0.000 *$ \\
\hline & SL & 0.16667 & -.08065 & .41398 & 1.483 & 0.166 \\
\hline & WA.physical & 0.16667 & -1.00907 & 1.34240 & .312 & 0.041 \\
\hline & WA.mental & -0.33333 & -2.05392 & 1.38726 & -.426 & 0.038 \\
\hline & FHP & 5.62917 & 4.00022 & 7.25811 & 7.606 & $0.000 *$ \\
\hline & $\mathrm{RSH}^{\top}$ & 6.40417 & 3.28478 & 9.52356 & 4.519 & $0.001 *$ \\
\hline & RB & 1.89583 & 0.16869 & 3.62298 & 2.416 & $0.034 *$ \\
\hline & UT - ave. & 0.09583 & 0.03523 & 0.15644 & 3.480 & $0.005^{\star}$ \\
\hline & UT - max. & 0.14750 & 0.05518 & 0.23982 & 3.517 & $0.005^{\star}$ \\
\hline & UT - onset & -119.333 & -468.56510 & 229.89843 & -.752 & 0.468 \\
\hline & UT - offset & 1408.000 & 242.71548 & 2573.2845 & 2.659 & $0.022^{*}$ \\
\hline & MT -ave. & -0.03417 & -0.14252 & 0.07418 & -0.694 & 0.502 \\
\hline & MT - max. & -0.02333 & -0.16929 & 0.12262 & -0.352 & 0.732 \\
\hline & MT - onset & -815.033 & -2099.1133 & 469.04668 & -1.397 & 0.190 \\
\hline & MT -offset & 1007.941 & -382.80656 & 2398.6899 & 1.595 & 0.139 \\
\hline & LT - ave. & -0.07750 & -0.19466 & 0.03966 & -1.456 & 0.173 \\
\hline & LT - max. & -0.12750 & -0.31262 & 0.05762 & -1.516 & 0.158 \\
\hline & LT - onset & -45.7166 & -396.91904 & 305.48571 & -0.287 & 0.780 \\
\hline & LT - offset & 910.8250 & 16.90960 & 1804.7404 & 2.243 & 0.056 \\
\hline & SA - ave. & -0.13667 & -0.24699 & -0.02634 & -2.726 & 0.020 * \\
\hline & SA - max. & -0.24250 & -0.42903 & -0.05597 & -2.861 & $0.015^{\star}$ \\
\hline & SA - onset & 48.42500 & -348.15777 & 445.00777 & 0.269 & 0.793 \\
\hline & SA - offset & 921.3416 & -280.81276 & 2123.4961 & 1.687 & 0.120 \\
\hline & SCM - ave. & 0.02167 & -0.07419 & 0.11752 & 0.497 & 0.629 \\
\hline & SCM -max. & 0.07250 & -0.10371 & 0.24871 & 0.906 & 0.385 \\
\hline & SCM - onset & -745.600 & -1872.3410 & 381.14100 & -1.456 & 0.173 \\
\hline
\end{tabular}




\begin{tabular}{|c|c|c|c|c|c|c|}
\hline & SCM - offset & 1053.433 & -148.57581 & 2255.4424 & 1.929 & 0.080 \\
\hline \multirow[t]{27}{*}{ Workplace } & Nsp & 2.50000 & 1.23606 & 3.76394 & 4.677 & $0.002 *$ \\
\hline & SL & 3.62500 & -2.40260 & 9.65260 & 1.422 & 0.198 \\
\hline & WA. physical & 0.50000 & -1.39592 & 2.39592 & 0.624 & 0.553 \\
\hline & WA.mental & -0.12500 & -1.70098 & 1.45098 & -0.188 & 0.857 \\
\hline & FHP & 3.82500 & 2.36547 & 5.28453 & 6.197 & $0.000 *$ \\
\hline & $\mathrm{RSH}$ & 3.30625 & .39228 & 6.22022 & 2.683 & $0.031^{*}$ \\
\hline & $\mathrm{RB}$ & 2.77500 & .98933 & 4.56067 & 3.675 & $0.008 *$ \\
\hline & UT - ave. & -0.01250 & -0.11766 & 0.09266 & -0.281 & 0.787 \\
\hline & UT - max. & 0.00375 & -0.15817 & 0.16567 & 0.055 & 0.958 \\
\hline & UT - onset & -206.287 & -592.17509 & 179.60009 & -1.264 & 0.247 \\
\hline & UT - offset & 660.7875 & -699.68739 & 2021.2623 & 1.149 & .288 \\
\hline & MT - ave. & -0.00375 & -0.01464 & 0.00714 & -0.814 & 0.442 \\
\hline & MT - max. & -0.02375 & -0.04159 & -0.00591 & -3.148 & 0.056 \\
\hline & MT - onset & 256.3250 & -401.93566 & 914.58566 & 0.921 & 0.388 \\
\hline & MT - offset & 25.63750 & -817.89258 & 869.16758 & .072 & 0.945 \\
\hline & LT -ave. & -0.03500 & -0.09611 & 0.02611 & -1.354 & 0.218 \\
\hline & LT -max. & -0.10000 & -0.24473 & 0.04473 & -1.634 & 0.146 \\
\hline & LT-onset & 545.0375 & -222.28843 & 1312.3634 & 1.680 & 0.137 \\
\hline & LT -offset & -117.875 & -1006.7134 & 770.96349 & -0.314 & 0.763 \\
\hline & SA - ave. & -0.11125 & -0.24945 & 0.02695 & -1.903 & 0.099 \\
\hline & SA - max. & -0.20500 & -0.46101 & 0.05101 & -1.893 & 0.100 \\
\hline & SA-onset & -155.600 & -546.49397 & 235.29397 & -.941 & 0.378 \\
\hline & SA-offset & -252.037 & -785.41698 & 281.34198 & -1.117 & 0.301 \\
\hline & SCM -ave. & -0.01000 & -0.18422 & 0.16422 & -0.136 & 0.896 \\
\hline & SCM -max. & 0.03125 & -0.27779 & 0.34029 & 0.239 & 0.818 \\
\hline & SCM - onset & -126.712 & -1304.8925 & 1051.4675 & -0.254 & 0.807 \\
\hline & SCM - offset & -180.812 & -2010.2942 & 1648.6692 & -0.234 & 0.822 \\
\hline
\end{tabular}


Table 3

Between-group differences (one-way ANCOVA) in NSP; SL; WA(physical/mental); FHP, RSH, RB angles; ave./ max. activity, onset/offset of UT,MT,LT,SA, and SCM.

\begin{tabular}{|c|c|c|c|c|c|c|}
\hline Variable & Group & Mean $\pm S D$ & $F$ & Sig. & $\begin{array}{l}\text { Partial } \\
\text { Eta } \\
\text { Squared }\end{array}$ & MCID \\
\hline \multirow[t]{3}{*}{ NSP } & Control & $4.27 \pm 1.34$ & 5.944 & $0.007 *$ & 0.306 & 0.975 \\
\hline & \multirow{2}{*}{$\begin{array}{l}\text { Online- } \\
\text { supervised }\end{array}$} & $3.25 \pm 1.95$ & & & & \multirow[t]{2}{*}{1.145} \\
\hline & & $4.12 \pm 2.29$ & & & & \\
\hline \multirow[t]{3}{*}{ SL } & Control & $1.45 \pm 2.73$ & 2.271 & 0.123 & 0.144 & 0.19 \\
\hline & $\begin{array}{l}\text { Online- } \\
\text { supervised }\end{array}$ & $0.16 \pm 0.38$ & & & & \multirow[t]{2}{*}{0.37} \\
\hline & Workplace & $0.37 \pm 0.74$ & & & & \\
\hline \multirow[t]{3}{*}{ WA.physical } & Control & $8.27 \pm .90$ & 0.780 & $0.048^{*}$ & 0.055 & 0.67 \\
\hline & \multirow{2}{*}{$\begin{array}{l}\text { Online- } \\
\text { supervised }\end{array}$} & $8.00 \pm 1.34$ & & & & \multirow[t]{2}{*}{1.22} \\
\hline & & $7.00 \pm 2.44$ & & & & \\
\hline \multirow[t]{3}{*}{ WA.mental } & Control & $7.63 \pm 1.56$ & 1.758 & $0.042^{\star}$ & 0.115 & 0.72 \\
\hline & \multirow{2}{*}{$\begin{array}{l}\text { Online- } \\
\text { supervised }\end{array}$} & $8.50 \pm 1.44$ & & & & \multirow[t]{2}{*}{1.12} \\
\hline & & $6.25 \pm 2.25$ & & & & \\
\hline \multirow{4}{*}{ FHP } & Control & $1510+80$ & 26762 & م0م0* & 0655 & 125 \\
\hline & \multirow{3}{*}{$\begin{array}{l}\text { Online- } \\
\text { supervised } \\
\text { Workplace }\end{array}$} & $3907+250$ & & & & \multirow{3}{*}{0.76} \\
\hline & & & & & & \\
\hline & & $41.01 \pm 1.02$ & & & & \\
\hline \multirow[t]{4}{*}{$\mathrm{RSH}$} & Control & $53.31 \pm 2.62$ & 10.046 & $0.001^{*}$ & 0.427 & 2.255 \\
\hline & \multirow{3}{*}{$\begin{array}{l}\text { Online- } \\
\text { supervised } \\
\text { Workplace }\end{array}$} & $46.93 \pm 4.51$ & & & & \multirow[t]{3}{*}{0.865} \\
\hline & & $49.91 \pm 1.73$ & & & & \\
\hline & & & & & & \\
\hline \multirow[t]{4}{*}{ RB } & Control & $50.78 \pm 3.65$ & 6.400 & $0.005^{\star}$ & 0.322 & 1.68 \\
\hline & \multirow{2}{*}{$\begin{array}{l}\text { Online- } \\
\text { supervised }\end{array}$} & $49.16 \pm 3.36$ & & & & \multirow[t]{3}{*}{1.63} \\
\hline & & $46.77 \pm 3.26$ & & & & \\
\hline & Workplace & & & & & \\
\hline
\end{tabular}




\begin{tabular}{|c|c|c|c|c|c|c|}
\hline Variable & Group & Mean $\pm S D$ & $F$ & Sig. & $\begin{array}{l}\text { Partial } \\
\text { Eta } \\
\text { Squared }\end{array}$ & MCID \\
\hline \multirow[t]{3}{*}{ UT - ave. } & Control & $0.28 \pm 0.13$ & 4.289 & $0.024^{\star}$ & 0.241 & 0.055 \\
\hline & \multirow{2}{*}{$\begin{array}{l}\text { Online- } \\
\text { supervised }\end{array}$} & $0.20 \pm 0.11$ & & & & \multirow[t]{2}{*}{0.075} \\
\hline & & $0.23 \pm 0.15$ & & & & \\
\hline \multirow[t]{3}{*}{ UT - max. } & Control & $0.44 \pm 0.19$ & 4.808 & $0.016^{\star}$ & 0.263 & 0.098635 \\
\hline & \multirow{2}{*}{$\begin{array}{l}\text { Online- } \\
\text { supervised }\end{array}$} & $0.3333 \pm 0.19727$ & & & & \multirow[t]{2}{*}{0.10724} \\
\hline & & $0.3600 \pm 0.21448$ & & & & \\
\hline \multirow[t]{4}{*}{ UT - onset } & Control & $-134.85 \pm 497.30$ & 0.789 & 0.464 & 0.055 & 99.205 \\
\hline & \multirow{2}{*}{$\begin{array}{l}\text { Online- } \\
\text { supervised }\end{array}$} & $-134.29 \pm 198.41$ & & & & \multirow[t]{3}{*}{175.73} \\
\hline & & $85.41 \pm 351.46$ & & & & \\
\hline & vrempiace & & & & & \\
\hline \multirow[t]{4}{*}{ UT - offset } & Control & $329.90 \pm 1093.61$ & 2.356 & 0.114 & 0.149 & 851.93 \\
\hline & \multirow{2}{*}{$\begin{array}{l}\text { Online- } \\
\text { supervised }\end{array}$} & $-762.35 \pm 1703.86$ & & & & \multirow[t]{3}{*}{910.015} \\
\hline & & $-1244.95 \pm 1820.03$ & & & & \\
\hline & Workplace & & & & & \\
\hline \multirow[t]{4}{*}{ MT - ave. } & Control & $0.17 \pm 0.14$ & 0.960 & 0.396 & 0.066 & 0.085 \\
\hline & \multirow{2}{*}{$\begin{array}{l}\text { Online- } \\
\text { supervised }\end{array}$} & $0.16 \pm 0.17$ & & & & \multirow[t]{3}{*}{0.035} \\
\hline & & $0.09 \pm 0.07$ & & & & \\
\hline & Workplace & & & & & \\
\hline \multirow[t]{3}{*}{ MT -max. } & Control & $0.35 \pm 0.31$ & 1.933 & 0.164 & 0.125 & 0.105 \\
\hline & \multirow{2}{*}{$\begin{array}{l}\text { Online- } \\
\text { supervised }\end{array}$} & $0.25 \pm 0.21$ & & & & \multirow[t]{2}{*}{0.075} \\
\hline & & $0.17 \pm 0.15$ & & & & \\
\hline \multirow{4}{*}{ MT - onset } & Control & $-77.27 \pm 307.90$ & 0.797 & 0.461 & 0.056 & 1039.285 \\
\hline & \multirow{2}{*}{$\begin{array}{l}\text { Online- } \\
\text { supervised }\end{array}$} & $501.47 \pm 2078.57$ & & & & \multirow[t]{3}{*}{197.455} \\
\hline & & $66.58 \pm 394.91$ & & & & \\
\hline & Workplace & & & & & \\
\hline
\end{tabular}




\begin{tabular}{|c|c|c|c|c|c|c|}
\hline Variable & Group & Mean $\pm S D$ & $F$ & Sig. & $\begin{array}{l}\text { Partial } \\
\text { Eta } \\
\text { Squared }\end{array}$ & MCID \\
\hline \multirow[t]{3}{*}{ MT - offset } & Control & $-346.70 \pm 2816.89$ & 1.152 & 0.331 & 0.079 & 1246.94 \\
\hline & $\begin{array}{l}\text { Online- } \\
\text { supervised }\end{array}$ & $-516.26 \pm 2493.89$ & & & & \multirow[t]{2}{*}{337.94} \\
\hline & Workplace & $-416.51 \pm 675.88$ & & & & \\
\hline \multirow[t]{3}{*}{ LT -ave. } & Control & $0.22 \pm 0.09$ & 0.314 & 0.733 & 0.023 & 0.09 \\
\hline & \multirow{2}{*}{$\begin{array}{l}\text { Online- } \\
\text { supervised }\end{array}$} & $0.25 \pm 0.18$ & & & & \multirow[t]{2}{*}{0.07} \\
\hline & & $0.18 \pm 0.15$ & & & & \\
\hline \multirow[t]{3}{*}{ LT -max. } & Control & $0.45 \pm 0.25$ & 0.200 & 0.820 & 0.015 & 0.155 \\
\hline & \multirow{2}{*}{$\begin{array}{l}\text { Online- } \\
\text { supervised }\end{array}$} & $0.44 \pm 0.31$ & & & & \multirow[t]{2}{*}{0.165} \\
\hline & & $0.40 \pm 033$ & & & & \\
\hline \multirow[t]{3}{*}{ LT -onset } & Control & $-74.22 \pm 333.85$ & 0.383 & 0.685 & 0.028 & 277.71 \\
\hline & \multirow{2}{*}{$\begin{array}{l}\text { Online- } \\
\text { supervised }\end{array}$} & $-105.75 \pm 555.42$ & & & & \multirow[t]{2}{*}{320.685} \\
\hline & & $-118.58 \pm 641.37$ & & & & \\
\hline \multirow{4}{*}{ LT -offset } & & & & & & \\
\hline & Control & $-101.27 \pm 1679.62$ & 0.762 & 0.477 & 0.053 & 790.62 \\
\hline & \multirow{2}{*}{$\begin{array}{l}\text { Online- } \\
\text { supervised }\end{array}$} & $-692.82 \pm 1581.24$ & & & & \multirow[t]{2}{*}{343.74} \\
\hline & & $-235.21 \pm 687.48$ & & & & \\
\hline \multirow[t]{3}{*}{ SA - ave. } & Control & $0.34 \pm 0.08$ & 0.096 & 0.908 & 0.007 & 0.07 \\
\hline & \multirow{2}{*}{$\begin{array}{l}\text { Online- } \\
\text { supervised }\end{array}$} & $0.34 \pm 0.14$ & & & & \multirow[t]{2}{*}{0.07} \\
\hline & & $0.32 \pm 0.14$ & & & & \\
\hline \multirow[t]{3}{*}{ SA - max. } & Control & $0.65 \pm 0.18$ & 0.014 & 0.986 & 0.001 & 0.11 \\
\hline & \multirow{2}{*}{$\begin{array}{l}\text { Online- } \\
\text { supervised }\end{array}$} & $0.63 \pm 0.22$ & & & & \multirow[t]{2}{*}{0.13} \\
\hline & & $0.64 \pm 0.26$ & & & & \\
\hline
\end{tabular}




\begin{tabular}{|c|c|c|c|c|c|c|}
\hline Variable & Group & Mean $\pm S D$ & F & Sig. & $\begin{array}{l}\text { Partial } \\
\text { Eta } \\
\text { Squared }\end{array}$ & MCID \\
\hline \multirow[t]{3}{*}{ SA - onset } & Control & $-152.12 \pm 497.08$ & 1.701 & 0.201 & 0.112 & 154.65 \\
\hline & \multirow{2}{*}{$\begin{array}{l}\text { Online- } \\
\text { supervised }\end{array}$} & $-218.33 \pm 309.30$ & & & & \multirow[t]{2}{*}{180.39} \\
\hline & & $101.58 \pm 360.78$ & & & & \\
\hline \multirow[t]{3}{*}{ SA - offset } & Control & $492.03 \pm 873.62$ & 0.885 & 0.424 & 0.062 & 928.275 \\
\hline & \multirow{2}{*}{$\begin{array}{l}\text { Online- } \\
\text { supervised }\end{array}$} & $-243.01 \pm 1856.55$ & & & & \multirow[t]{2}{*}{315.895} \\
\hline & & $210.45 \pm 631.79$ & & & & \\
\hline \multirow[t]{4}{*}{ SCM -ave. } & Control & $0.27 \pm 0.18$ & 0.490 & 0.618 & 0.035 & 0.11 \\
\hline & \multirow{2}{*}{$\begin{array}{l}\text { Online- } \\
\text { supervised }\end{array}$} & $0.29 \pm 0.22$ & & & & \multirow[t]{3}{*}{0.12} \\
\hline & & $0.34 \pm 0.24$ & & & & \\
\hline & Workplace & & & & & \\
\hline \multirow[t]{4}{*}{ SCM - max. } & Control & $0.49 \pm 0.32$ & 0.820 & 0.451 & 0.057 & 0.155 \\
\hline & \multirow{2}{*}{$\begin{array}{l}\text { Online- } \\
\text { supervised }\end{array}$} & $0.46 \pm 0.31$ & & & & \multirow[t]{3}{*}{0.165} \\
\hline & & $0.48 \pm 0.33$ & & & & \\
\hline & Workplace & & & & & \\
\hline \multirow[t]{3}{*}{ SCM -onset } & Control & $284.38 \pm 716.78$ & 0.383 & 0.685 & 0.028 & 914.44 \\
\hline & \multirow{2}{*}{$\begin{array}{l}\text { Online- } \\
\text { supervised }\end{array}$} & $783.87 \pm 1828.88$ & & & & \multirow[t]{2}{*}{386.195} \\
\hline & & $627.11 \pm 772.39$ & & & & \\
\hline \multirow{3}{*}{$\begin{array}{l}\text { SCM - } \\
\text { offset }\end{array}$} & Control & $-395.87 \pm 1322.97$ & 0.708 & 0.501 & 0.050 & 1075.325 \\
\hline & \multirow{2}{*}{$\begin{array}{l}\text { Online- } \\
\text { supervised }\end{array}$} & $-1152.22 \pm 2150.65$ & & & & \multirow[t]{2}{*}{801.995} \\
\hline & & $-712.12 \pm 1603.99$ & & & & \\
\hline
\end{tabular}

\section{Discussion}

The present study found that online-supervised corrective exercises seem to be effective in improving a range of parameters related to work performance. These findings are highly relevant in the light of the ongoing COVID pandemic, where many workers have to work from home.

The findings confirmed that performing eight-week corrective exercises had a significant effect on the NSP among the subjects in the two intervention groups. This is in line with previous findings showing 
that corrective exercises improve the neck pain (Mehri, Letafatkar et al. 2020). Many studies reported that prolonged sitting or improper neck and shoulder posture during the work, may have some association with NSP among the office workers (Lee, Lee et al. 2017). Neck pain is associated with the ability to maintain an upright posture, as reported previously, and there could be a relationship among the correction of forward head posture, round shoulders, and pain (Lynch, Thigpen et al. 2010). In this regard, Lee et al. suggested that maintaining an upright body position and implementing posture-correction strategies in different sitting positions, can prevent musculoskeletal pain (Cools, Struyf et al. 2014, Lee, Lee et al. 2017). Hence, all the subjects were initially informed about the UCS consequences; hence, their postural awareness was repeatedly developed throughout the study days and frequent corrections to upright neutral postural' position were recommended focusing on the control system in postural reeducation programs such as memory joggers, to ensure that those behaviors become kinds of habit (Abdollahzade, Shadmehr et al. 2017).

Further, some researchers recommended the muscle strengthening or endurance exercises for an incorrect posture leading to pain through increased load on the cervical spine and changing the muscle's length (Gram, Holtermann et al. 2012). Meanwhile, training and rehabilitation of power and endurance of the deep neck flexors, have been previously proved to be beneficial for restoring neuromuscular imbalance and cervical stabilization; where, the present study highlighted relevant exercises (Falla, Jull et al. 2007, Lee, Kim et al. 2018). Considering that the scapula plays an important role in NSP and the keystone of the UCS, the current study primarily focused on the function of the scapula muscles to restore and balance the scapula stabilizers. This phenomenon could be due to activating the neck muscles and reducing the compensatory movements of the muscles involved in the area (Cagnie, Struyf et al. 2014, Cools, Struyf et al. 2014, Kang, Im et al. 2021).

Our findings significantly affected the postural angles. Since the alteration in the resting scapular position may occur with the abnormal alignment of cervical and thoracic spine, the forward head posture can result in round shoulders, which may cause enhancing thoracic kyphotic posture, respectively(Kwon, Son et al. 2015). Based on the literature, there might be a relationship among the forward head posture, round shoulders, and round back, with the studies revealing an improvement in response to training programs' exercises (Sheikhhoseini, Shahrbanian et al. 2018). It is supposed that the involved muscles in the UCS may be shortened or lengthened when they are remained in the improper posture for a long time, occurring adaptive lengthening or shortening over the time leading to poor posture. The traditional approach has focused on improving malalignments by stretching the shortened and strengthening the weakened muscles. However, using stretching exercises, some studies reported no changes in the muscle length but indicated improved tolerance to stretching (Sahrmann, Azevedo et al. 2017).

In contrast, the functional approach states that the interactions among all central and peripheral nervous systems, muscular and skeletal structures are the cause of WMSDs (Hamill, van Emmerik et al. 1999). Thus, the initial strategy containing posture, muscle activation, and movement pattern intervention is recommended to be more effective (Hodges, Van Dieën et al. 2019). It seems that focusing simultaneously on the neural and muscular components for improving postural malalignments, brings 
about the best results if the intervention is applied at least six weeks with an average of three times a week, and duration of 15 to 60 minutes (Bayattork, Sköld et al. 2020).

The upper trapezius significantly improved in the current study; while, no alterations were found in others (33). The results are to seme extent in line with the previous findings of Arshadi and et al., conducted among healthy men with the UCS. In their experimet they reported that an eight-week corrective exercise exhibits significant differences in EMG activity of sternocleidomastoid, upper trapezius, serratus anterior; though, middle and lower trapezius activity revealed no changes (Arshadi, Ghasemi et al. 2019).

Meanwhile, Seidi et al. detected significant differences in middle, lower trapezius, and serratus anterior activation levels performing a comprehensive corrective exercise program by the young males with no pain; nevertheless, the muscle activation timing indicated no specific differences. In the current study, upper trapezius offset timing varied in the online- supervised group (Seidi, Bayattork et al. 2020). Concerning the muscle activation timing, Cools et al. supported the theory that the individuals with pain may have delayed onset of middle and lower trapezius muscle activity, and injured individuals show abnormal muscle recruitment patterns in the same muscles. The timing of muscle activation is an essential factor in coordination with the scapula and arm movement (Cools, Witvrouw et al. 2003).

Due to the presence of a neuromuscular imbalance in the UCS needing sensory-motor function, muscle adaptation to resistance training could happen before the development of hypertrophy, influencing more motor units, learning to use active motor units more effectively, and reducing the inhibitory inputs of alpha motor neurons (Hakkinen 1994).

In our study, the upper trapezius was the only muscle, which displayed activation levels' decrease. This outcome was is in line with the findings of the studies that evaluated the upper trapezius activity among the office workers. It is worth noting that the muscle activation can oocure by the neural adaptations associated with short-term exercise or may be connected with reduced pain levels rather than a direct result of the exercise program (Villanueva, Rabal-Pelay et al. 2020). In this regard, Andersen et al. described these phenomena in their "wheel of pain reduction" and concluded that specific strength after exercise relieves pain and maximizes the activity, particularly in painful trapezius muscles. Increasing shoulder abduction strength in women with trapezius myalgia and reducing relative workload may indirectly decrease pain (Andersen, Andersen et al. 2009). Consequently, the diminished upper trapezius activation levels prove this issue as the only trapezius muscle part, and often overactivate in the subjects with UCS; so, its lesser activation might lead to pain relief (Kwon, Son et al. 2015).

During this research, the applied intervention improved mental and physical workability in the onlinesupervised group, which was consistent with the study of Gram et al. However, no statistical differences were found for the sick-leave as a fundamental problem when the individuals are going to work with pain (Gram, Holtermann et al. 2012). Musculoskeletal pain may negatively affect the workability, and physical activity and could make significant differences concerning sedentary behavior, work absenteeism, and workability, which might be associated with NSP (Hallman, Holtermann et al. 2019). 


\section{Limitations}

Intervention duration in the current study can be considered as a limitation, since UCS influencing scapular muscles' activation may need more time to achieve the appropriate recruitment, timing, and firing rates. Although, postural alignment and pain improvements occurr faster, the upper trapezius has started changes. Thus, more comprehensive studies are required to manage the UCS as a sensory-motor disorder due to neuromuscular adaptation. Another limitation of current studywas relatively small sample size, which was selected to recruite the subjects in COVID 19 conditions. Although, the study had sufficient statistical capability to show clear differences between corrective exercises and the control conditions, it was not enough powerful to compare the two active interventions. However, the main finding of the present study - i.e. that online-supervised corrective exercises are effective - remains relevant to similar types of subjects with UCS. Eventually, lack of blinding in the study design could be assumed as another unachievable limitation with the exercise interventions.

\section{Conclusion}

Implementing an eight-week corrective exercises' program resulted in clinically desired significant improvements in most study outcomes. The findings in the online supervised group revealed significant changes, which confirm that the supervised intervention could be more effective than un-supervised intervention. However, it seems very difficult to compare the consequences of the current study with those of others. The reason is that previous research with similar titles, evaluated the effects of various exercises on different study populations with different outcome measures, which make it difficult to interpret the results. In the present study, the magnitude of the effect sizes in intervention groups, indicated that prescribed corrective exercises, positively affect NSP, forward head posture, round shoulders, round back, workability and restored upper trapezius muscle activity. Based on the findings, it is suggested to perform the training under direct supervision rather than alone and without any suppervision. Further, WMSDs impose indirect costs, including productivity loss, treatment costs, and disability. Hence, managing the UCS is highly recommended among the office workers, particularly the computer users with NSP in order to increase health condition and early prevention in the workplace, which in turn could significantly increase the workability. However, it is worth mentioning that noting is worthwhile to see the individuals work hard but always experience pain.

\section{Declarations}

\section{Funding}

The authors declare that no funds, grants, or other support were received during the preparation of this manuscript.

\section{Competing Interests}


Authors Zohreh Yaghoubitajani(ZY), Mehdi Gheitasi(MG), Mohammad Bayattork(MB), and Lars Louis Andersen(LLA) declare that they have no relevant financial or non-financial interests to disclose.

\section{Acknowledgments}

We would like to thank the authors' team members for their contributions to the success of this trial.

\section{Authors Contributions}

ZY and MG contributed to the study conception and design. ZY performed clinical examination and data collection. MG and MB participated in the methodologically development and design of the statistical analysis. ZY wrote the first draft of the manuscript. LLA contributed to the comments and suggestions that greatly improved the manuscript. Finally, all the authors revised it critically for important intellectual content, agreed with the content, contributed to the current study's refinement, and approved the final manuscript.

\section{Data Availability}

The datasets generated during and/or analysed during the current study are available from the corresponding author on reasonable request. The researchers interested in using the final dataset for scientific purposes may contact the corresponding author.

\section{Ethics approval}

This study was performed in line with the principles of the Declaration of Helsinki. Approval was granted by Ethics Committee on the Research at SBU, Tehran, Iran (approval number: IR.SBU.REC.1399.036 / 2020.06.20)

\section{Consent to publish}

Not applicable

\section{Conflict of interest}

Authors Zohreh Yaghoubitajani(ZY), Mehdi Gheitasi(MG), Mohammad Bayattork(MB), and Lars Louis Andersen(LLA) declare that they have no conflict of interest.

\section{Informed consent}

All procedures followed were in accordance with the ethical standards of the responsible committee on human experimentation (institutional and national) and with the Helsinki Declaration of 1975, as revised in 2000 (5). Informed consent was obtained from all the individual subjects for being included in the study.

\section{References}


1. Abdollahzade Z, Shadmehr A, Malmir K, Ghotbi N (2017) Effects of 4 week postural corrective exercise on correcting forward head posture. Journal of Modern Rehabilitation 11(2):85-92

2. Aegerter AM, Deforth M, Sjøgaard G, Johnston V, Volken T, Luomajoki H, Dratva J, Dressel H, Distler O, Melloh M (2021) "No evidence for a decrease in physical activity among swiss office workers during Covid-19: a longitudinal study." Frontiers in psychology 12

3. Andersen JH, Harhoff M, Grimstrup S, Vilstrup I, Lassen CF, Brandt LP, Kryger Al, Overgaard E, Hansen KD, Mikkelsen S (2008) Computer mouse use predicts acute pain but not prolonged or chronic pain in the neck and shoulder. Occup Environ Med 65(2):126-131

4. Andersen LL, Andersen JL, Suetta C, Kjær M, Søgaard K, Sjøgaard G (2009) Effect of contrasting physical exercise interventions on rapid force capacity of chronically painful muscles. J Appl Physiol 107(5):1413-1419

5. Arshadi R, Ghasemi GA, Samadi H (2019) Effects of an 8-week selective corrective exercises program on electromyography activity of scapular and neck muscles in persons with upper crossed syndrome: Randomized controlled trial. Phys Ther Sport 37:113-119

6. Bayattork M, Jakobsen MD, Sundstrup E, Seidi F, Bay H, Andersen LL (2019) "Musculoskeletal pain in multiple body sites and work ability in the general working population: cross-sectional study among 10,000 wage earners". Scandinavian journal of pain 19(1):131-137

7. Bayattork M, Sköld MB, Sundstrup E, Andersen LL (2020) Exercise interventions to improve postural malalignments in head, neck, and trunk among adolescents, adults, and older people: systematic review of randomized controlled trials. Journal of exercise rehabilitation 16(1):36

8. Blangsted AK, Søgaard K, Hansen EA, Hannerz H, Sjøgaard G (2008) "One-year randomized controlled trial with different physical-activity programs to reduce musculoskeletal symptoms in the neck and shoulders among office workers."Scandinavian journal of work, environment \& health:5565

9. Brandt M, Sundstrup E, Jakobsen MD, Jay K, Colado JC, Wang Y, Zebis MK, Andersen LL (2014) "Association between neck/shoulder pain and trapezius muscle tenderness in office workers." Pain research and treatment 2014

10. Cagnie B, Struyf F, Cools A, Castelein B, Danneels L, O'leary S (2014) The relevance of scapular dysfunction in neck pain: a brief commentary. journal of orthopaedic \& sports physical therapy 44(6):435-439

11. Cools AM, Struyf F, De Mey K, Maenhout A, Castelein B, Cagnie B (2014) Rehabilitation of scapular dyskinesis: from the office worker to the elite overhead athlete. Br J Sports Med 48(8):692-697

12. Cools AM, Witvrouw EE, Declercq GA, Danneels LA, Cambier DC (2003) "Scapular muscle recruitment patterns: trapezius muscle latency with and without impingement symptoms". Am J Sports Med 31(4):542-549

13. Coury HJ, Moreira RF, Dias NB (2009) "Efetividade do exercício físico em ambiente ocupacional para controle da dor cervical, lombar e do ombro: uma revisão sistemática". Braz J Phys Ther 13:461-479 
14. D'Anna C, Schmid M, Conforto S (2021) Linking head and neck posture with muscular activity and perceived discomfort during prolonged smartphone texting. Int J Ind Ergon 83:103134

15. De Mey K, Danneels L, Cagnie B, Cools AM (2012) "Scapular muscle rehabilitation exercises in overhead athletes with impingement symptoms: effect of a 6-week training program on muscle recruitment and functional outcome". Am J Sports Med 40(8):1906-1915

16. Falla D, Jull G, Russell T, Vicenzino B, Hodges $P$ (2007) "Effect of neck exercise on sitting posture in patients with chronic neck pain". Phys Ther 87(4):408-417

17. Falla DL, Jull GA, Hodges PW (2004) Patients with neck pain demonstrate reduced electromyographic activity of the deep cervical flexor muscles during performance of the craniocervical flexion test. Spine 29(19):2108-2114

18. Gram B, Andersen C, Zebis MK, Bredahl T, Pedersen MT, Mortensen OS, Jensen RH, Andersen LL, Sjøgaard G (2014) "Effect of training supervision on effectiveness of strength training for reducing neck/shoulder pain and headache in office workers: cluster randomized controlled trial." BioMed research international 2014

19. Gram B, Holtermann A, Bültmann U, Sjøgaard G, Søgaard K (2012) Does an exercise intervention improving aerobic capacity among construction workers also improve musculoskeletal pain, work ability, productivity, perceived physical exertion, and sick leave?: a randomized controlled trial. J Occup Environ Med 54(12):1520-1526

20. Hakkinen K (1994) Neuromuscular adaptation during strength training, ageing, detraining, and immobilization. Critical Reviews in Physical and Rehabilitation Medicine 14:161-198

21. Hallman DM, Holtermann A, Dencker-Larsen S, Jørgensen MB, Rasmussen CDN (2019) Are trajectories of neck-shoulder pain associated with sick leave and work ability in workers? A 1-year prospective study. BMJ open 9(3):e022006

22. Hamill J, van Emmerik RE, Heiderscheit BC, Li L (1999) "A dynamical systems approach to lower extremity running injuries". Clin Biomech Elsevier Ltd 14(5):297-308

23. Helgadottir H, Kristjansson E, Einarsson E, Karduna A, Jonsson H Jr (2011) Altered activity of the serratus anterior during unilateral arm elevation in patients with cervical disorders. J Electromyogr Kinesiol 21(6):947-953

24. Hodges P, PhD, DSc MedDr, Paul BPhty, Van Dieën WJH, Cholewicki J (2019) Time to reflect on the role of motor control in low back pain. JOSPT, Inc. JOSPT. 1033 North Fairfax Street, Suite 304, Alexandria, VA

25. Isper Garbin AJ, Soares GB, Arcieri RM, Saliba Garbin CA, Siqueira CE (2017) "Musculoskeletal disorders and perception of working conditions: A survey of Brazilian dentists in São Paulo." International journal of occupational medicine and environmental health:367-377

26. Johnson G, Bogduk N, Nowitzke A, House D (1994) Anatomy and actions of the trapezius muscle. Clin Biomech Elsevier Ltd 9(1):44-50

27. Johnston V, Jull G, Souvlis T, Jimmieson NL (2008) "Neck movement and muscle activity characteristics in female office workers with neck pain. " Spine 33(5):555-563 
28. Kang N-Y, Im S-C, Kim K (2021) "Effects of a combination of scapular stabilization and thoracic extension exercises for office workers with forward head posture on the craniovertebral angle, respiration, pain, and disability: A randomized-controlled trial."

29. Karimian R, Rahnama N, Ghasemi G, Lenjannejadian S (2019) Photogrammetric Analysis of Upper Cross Syndrome among Teachers and the Effects of National Academy of Sports Medicine Exercises with Ergonomic Intervention on the Syndrome. Journal of research in health sciences 19(3): e00450

30. Kwon JW, Son SM, Lee NK (2015) Changes in upper-extremity muscle activities due to head position in subjects with a forward head posture and rounded shoulders. Journal of physical therapy science 27(6):1739-1742

31. Lee J, Kim D, Yu K, Cho Y, You JH (2018) Comparison of isometric cervical flexor and isometric cervical extensor system exercises on patients with neuromuscular imbalance and cervical crossed syndrome associated forward head posture. Biomed Mater Eng 29(3):289-298

32. Lee $S$, Lee $Y$, Chung $Y$ (2017) Effect of changes in head postures during use of laptops on muscle activity of the neck and trunk. Physical therapy rehabilitation science 6(1):33-38

33. Lynch SS, Thigpen CA, Mihalik JP, Prentice WE, Padua D (2010) The effects of an exercise intervention on forward head and rounded shoulder postures in elite swimmers. Br J Sports Med 44(5):376-381

34. Madeleine P, Lundager B, Voigt M, Arendt-Nielsen L (1999) Shoulder muscle co-ordination during chronic and acute experimental neck-shoulder pain. An occupational pain study. Eur J Appl Physiol Occup Physiol 79(2):127-140

35. Mehri A, Letafatkar A, Khosrokiani Z (2020) Effects of Corrective Exercises on Posture, Pain, and Muscle Activation of Patients With Chronic Neck Pain Exposed to Anterior-Posterior Perturbation. J Manip Physiol Ther 43(4):311-324

36. Nejati P, Lotfian S, Moezy A, Moezy A, Nejati M (2014) The relationship of forward head posture and rounded shoulders with neck pain in Iranian office workers. Medical journal of the Islamic Republic of Iran 28:26

37. Norman GR, Sloan JA, Wyrwich KW (2003) "Interpretation of changes in health-related quality of life: the remarkable universality of half a standard deviation."Medical care:582-592

38. Ohlendorf D, Erbe C, Nowak J, Hauck I, Hermanns I, Ditchen D, Ellegast R, Groneberg DA (2017) Constrained posture in dentistry-a kinematic analysis of dentists. BMC Musculoskelet Disord 18(1):1-15

39. Page P, Frank CC, Lardner R (2010) Assessment and treatment of muscle imbalance: the Janda approach, Human kinetics

40. Richardson JT (2011) Eta squared and partial eta squared as measures of effect size in educational research. Educational research review 6(2):135-147

41. Sahrmann S, Azevedo DC, Van Dillen L (2017) Diagnosis and treatment of movement system impairment syndromes. Braz J Phys Ther 21(6):391-399 
42. Seidi F, Bayattork M, Minoonejad H, Andersen LL, Page P (2020) "Comprehensive corrective exercise program improves alignment, muscle activation and movement pattern of men with upper crossed syndrome: randomized controlled trial". Sci Rep 10(1):1-11

43. Sheikhhoseini R, Shahrbanian S, Sayyadi P, O'Sullivan K (2018) Effectiveness of therapeutic exercise on forward head posture: a systematic review and meta-analysis. J Manip Physiol Ther 41(6):530539

44. Singla D, Veqar Z (2017) "Association Between Forward Head, Rounded Shoulders, and Increased Thoracic Kyphosis: A Review of the Literature". J Chiropr Med 16(3):220-229

45. Skamagki G, King A, Duncan M, Wåhlin C (2018) A systematic review on workplace interventions to manage chronic musculoskeletal conditions. Physiotherapy Research International 23(4):e1738

46. Sundstrup E, Seeberg KGV, Bengtsen E, Andersen LL (2020) A systematic review of workplace interventions to rehabilitate musculoskeletal disorders among employees with physical demanding work. J Occup Rehabil 30(4):588-612

47. Szeto GP, Straker LM, O'Sullivan PB (2005) A comparison of symptomatic and asymptomatic office workers performing monotonous keyboard work -1 : neck and shoulder muscle recruitment patterns. Man Therap 10(4):270-280

48. Villanueva A, Rabal-Pelay J, Berzosa C, Gutiérrez H, Cimarras-Otal C, Lacarcel-Tejero B, BatallerCervero AV (2020) Effect of a long exercise program in the reduction of musculoskeletal discomfort in office workers. Int J Environ Res Public Health 17(23):9042

49. Yaghoubitajani Z, Gheitasi M, Bayattork M, Andersen LL (2021) Online supervised versus workplace corrective exercises for upper crossed syndrome: a protocol for a randomized controlled trial. Trials 22(1):907

50. Zad SS, Patil P (2021) Effectiveness of Janda's Approach in Upper Cross syndrome in Medical Students. Annals of the Romanian Society for Cell Biology 25(6):17385-17399

\section{Figures}




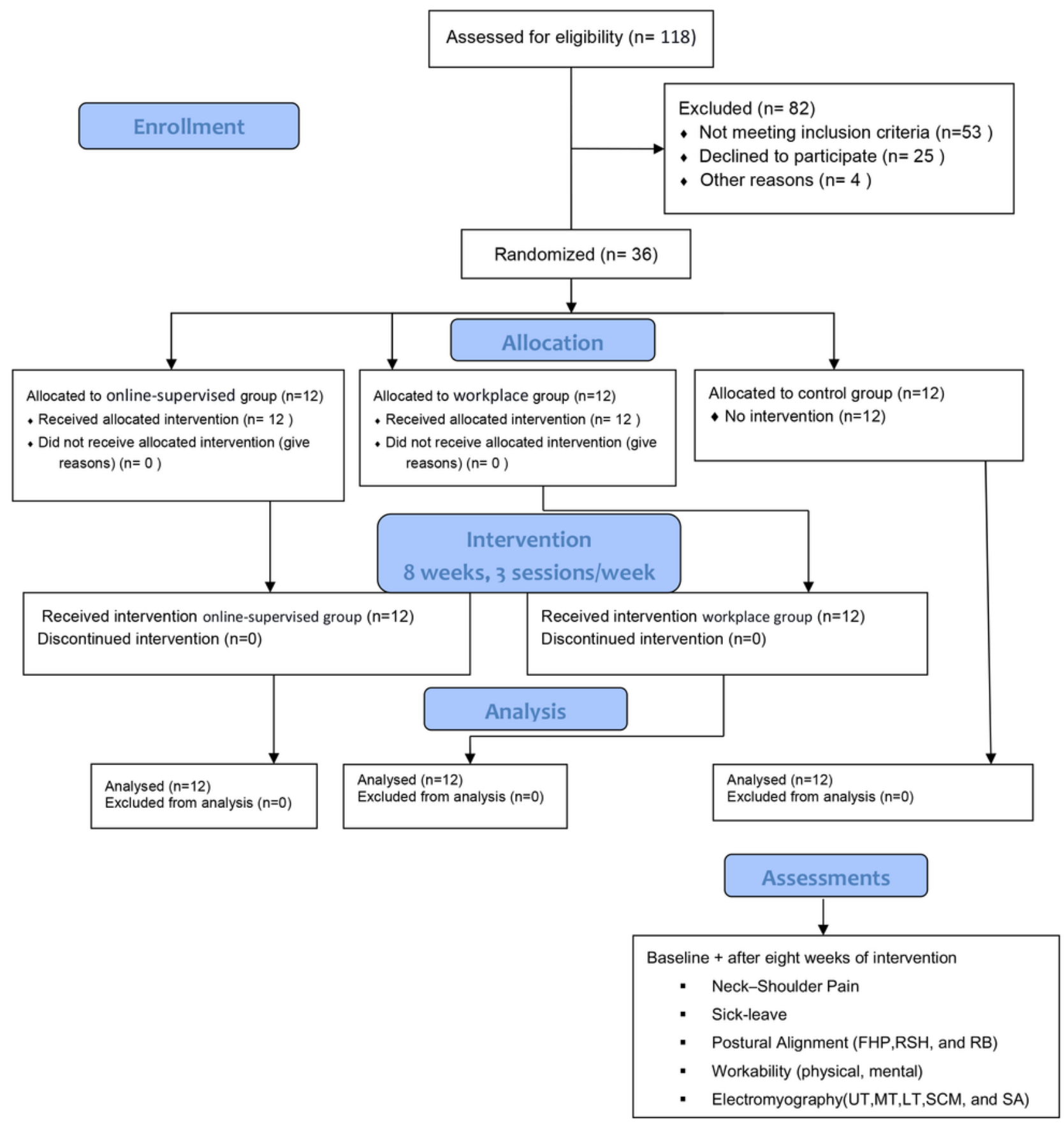

\section{Figure 1}

Study flowchart

\section{Supplementary Files}

This is a list of supplementary files associated with this preprint. Click to download. 
- CONSORTChecklist.doc

Page 25/25 